\title{
Evaluating Cerebral Perfusion in Alzheimer Patients and First-Degree Relatives: Lessons from Artemisa Province, Cuba
}

\author{
Yamilé Peña-Quián MD PhD, Saily Sosa-Pérez MD MS, Juan F. Batista-Cuéllar MD, Chryslaine Rodríguez-Tanty PhD, \\ Leonel A. Torres-Aroche PhD, Elvia Luz-Sánchez RN MS, Susana Romero-Collado RN MS
}

\begin{abstract}
INTRODUCTION Alzheimer disease is related to several risk factors including aging, family history, high blood pressure and diabetes. Studies have shown specific regional cerebral perfusion changes in patients with Alzheimer disease. Some authors state that these changes could appear years before patient memory becomes impaired, enabling early diagnosis in high-risk persons who appear to be healthy.

OBJECTIVE Determine the usefulness of cerebral perfusion studies in Alzheimer patients and first-degree relatives for obtaining additional diagnostic information and detecting functional changes that may suggest elevated disease risk.

METHODS This study involved 128 persons (87 clinically diagnosed with Alzheimer disease and 41 of their first-degree relatives with normal cognition), all from Artemisa Province, Cuba. We performed clinical, laboratory, neuropsychological and genetic (apolipoprotein $\mathrm{E}-\mathrm{ApoE}$, e4 allele) tests, as well as cerebral perfusion studies using single photon emission computed tomography after administering $740-925 \mathrm{MBq}$ of $99 \mathrm{~m}$ Tc-ECD, following internationally standardized protocols.
\end{abstract}

RESULTS In the Alzheimer disease group, the cerebral single photon emission computed tomography showed a typical Alzheimer pattern

\section{INTRODUCTION}

Alzheimer disease (AD) is a neurodegenerative condition with insidious onset and slow progression. The disease is responsible for $70 \%$ of dementia cases and is the main cause of dementia in persons aged $\geq 65$. [1]

The main risk factors for onset of the disease are age, family history of $A D$, high blood pressure, high cholesterol, obesity, diabetes mellitus and low education level. The disease is more common in women and has been linked to the e 4 allele of the gene that codes for ApoE.[2]

Longer life expectancy leads to an older population and increases in $A D$ incidence. Cuba has one of the oldest populations in Latin America.[3] According to the annual Cuban Health Statistics Report, in 2019 the Cuban population

\section{IMPORTANCE}

This study confirms the usefulness of cerebral perfusion single photon emission computed tomography in evaluating patients with different stages of Alzheimer disease and their first-degree relatives. In first-degree relatives, diagnosing abnormalities may allow for early diagnosis of the disease and possible early interventions in the community. (bilateral posterior temporal-parietal hypoperfusion) in $77 \%(67 / 87)$ of participants; $35.9 \%(28 / 67)$ in stage $1 ; 51.3 \%(40 / 67)$ in stage 2; and $12.8 \%(10 / 67)$ in stage 3 of the disease. In this group, $12.7 \%$ $(11 / 87)$ had mild or unilateral cerebral perfusion changes; $5.7 \%(5 / 87)$ vascular dementia; $3.4 \%(3 / 87)$ frontal dementia; and $1.2 \%(1 / 87)$ normal cerebral perfusion. Of the patients, $28.7 \%(25 / 87)$ received a different classification of stage and disease diagnosis after cerebral perfusion results were considered. In the relative group, $14.6 \%(6 / 41)$ had cerebral perfusion abnormalities. Among these, $7.1 \%(3 / 41)$ were mild bilateral temporal-parietal hypoperfusion; $4.8 \%(2 / 41)$ mild unilateral temporal-parietal hypoperfusion; and $2.4 \%(1 / 41)$ had perfusion defecits in their right frontal lobes. Of patients with typical Alzheimer disease patterns in the cerebral single photon emission computed tomography, $76.6 \%$ (52/67) had positive ApoE e4. All relatives with perfusion abnormalities (6/6) had positive ApoE e4.

CONCLUSIONS Cerebral perfusion studies confirmed the Alzheimer disease diagnosis, classified disease stages, and differentiated between the types of dementia. The test showed perfusion changes in several asymptomatic first-degree relatives with positive ApoE e4, which could be predictors of disease. The technique was useful for evaluating patients and their relatives.

KEYWORDS Cerebrovascular circulation; tomography, emissioncomputed, single-photon; Alzheimer disease; Alzheimer's disease; Cuba

was $11,201,549$, with an average life expectancy of 78.4 years. The number of persons aged $\geq 60$ has doubled in less than 40 years and comprised $20 \%$ of the population.[3] It is estimated that in 2025, 1 in every 4 persons will be aged $\geq 60$ and about 500,000 will be aged $\geq 80$. [2]

Dementia is the sixth leading cause of death in Cuba.[3] In 2019, dementia was the cause of death in 5097 persons, with a gross rate of 45.4 and an adjusted rate of 16.7 per 100,000 population.[3] A 2012 prospective longitudinal study in Havana and Matanzas Provinces showed an annual dementia incidence of 21.2 per 100,000 population.[4] Dementia prevalence is $6.4 \%-10.2 \%$ in persons aged $\geq 65$ years, and is more common in women. In the 2012 study, AD was the most common cause of dementia.[4] Considering the rapid aging of the Cuban population, it is estimated that 130,000 persons would have AD or another type of dementia by $2025 .[4]$

$A D$ is one of the main causes of disability in older adults and also a disability that leads to the greatest dependence, financial burden and psychological stress on caregivers. [5] Without an effective intervention, by 2050 the number of new dementia cases in persons aged $\geq 65$ will substantially increase yearly costs. The long-term demands on caregivers of persons with dementia will increase far beyond current levels, with significant social and economic impacts.[2,6,7] 
Diagnosing $A D$ is primarily a clinical endeavor and based on a triad of medical history, caregiver-provided information and cognitive testing. With current technological advances, structural biomarkers, biological biomarkers and nuclear neuroimaging have been added to confirmatory diagnostic criteria.[8-10] In summary, the criteria are:

Hippocampal amnesia (alone or associated with other cognitive or behavioral impairments) appearing at any age, with insidious onset and progressive worsening, associated with one or more of the following biomarkers:

- Structural: Medial temporal lobe atrophy (detected by MRI)

- Biological: Abnormalities in cerebrospinal fluid biomarkers

- Functional or metabolic: Regional cerebral hypoperfusion detected by single photon emission computed tomography (SPECT), fluorodeoxyglucose hypometabolism and observation of amyloid or tau protein buildups using positron emission tomography (PET).

The new diagnostic criteria show that AD's physiopathological process starts several years before the first clinical symptoms appear. These criteria support the use of biomarkers that can predict $A D$ progression in preclinical phases and support diagnosis in stages of mild cognitive impairment or dementia. While some biomarkers are currently used for research purposes, they may also be available in clinical practice in the near future.[9]

SPECT is a minimally invasive technique that plays a fundamental role in brain function assessment in establishing AD diagnosis, disease stage, prognosis, differential diagnosis and evaluation of treatment options for $A D$ patients.[11,12] Patients receive an intravenous radiopharmaceutical that crosses the blood-brain barrier, permitting SPECT gamma cameras to acquire 3D images.[11,12]

AD patients show specific changes in cerebral perfusion diagnosed by SPECT.[13] In stage 1 (disease onset), there tends to be mild perfusion changes in the cerebral cortex, which can be unilateral and localized. As the disease progresses to stage 2, these changes form a typical bilateral posterior temporal-parietal hypoperfusion pattern. In advanced stages, hypoperfusion can extend to the frontal lobes of the cerebral cortex. Hypoperfusion of the posterior cingulate can also occur.[13] In AD, perfusion remains normal in the sensory-motor cortex, basal ganglia, cerebellum and brainstem. These patterns, along with clinical manifestations and other test results, allow for evaluation and differential diagnosis of the types of dementia.[13]

In practice, diagnosing dementia continues to be a clinical endeavor, which substantially contributes to delayed diagnoses because once symptoms manifest, the neuropathological abnormalities are generally already extensive. This leads to failure of current drug and non-drug therapies. The fundamental role of and the challenge in developing new neuroimaging techniques is being able to detect with a high degree of sensitivity and specificity the predictive functional abnormalities that lead to irreversible structural damage.[14]

Some authors suggest that these functional abnormalities could appear in persons many years before memory impairment. $[8,14,15]$ In high-risk populations, tests such as SPECT could lead to early diagnosis, allowing for timely intervention before irreversible memory loss occurs. Lopera reported on SPECT's value in evaluating patients' relatives.[16] However, research is still insufficient.

Detecting abnormality patterns in functional neuroimaging in asymptomatic carriers of familial Alzheimer disease could serve as an early diagnostic marker before the age of onset in subjects at risk of sporadic AD.[16]

Cuba's Ministry of Public Health has been working for years to develop stratified active screening programs at all three levels of health care-primary, secondary and tertiary. These programs could be optimized with neuroimaging.[17] Yet the usefulness of neuroimaging has not been defined in our setting, and it is not widely used by specialists charged with AD patient care.

The objective of this study is to determine the usefulness of the SPECT cerebral perfusion test in $A D$ patients and their firstdegree relatives in obtaining additional diagnostic information and detecting functional changes that suggest elevated disease risk.

\section{METHODS}

Type of study and participants We conducted an observational, analytic cohort study from January 2018 through March 2020. 128 participants were seen at the Comprehensive Memory Disorder Community Care Service in San Antonio de los Baños, Artemisa Province, Cuba, and were screened based on criteria described below. We conducted a medical interview, clinical examination, laboratory tests (complete blood count, blood glucose, urea, creatinine and lipid panel), neuropsychological evaluation, ApoE e4 test, non-contrasted CT scan, and cerebral perfusion SPECT. The SPECT was conducted at the Isotope Center's Clinical Research Division in Havana.

We divided participants into two groups: AD patients (87 with a clinical diagnosis of $A D$ ) and their first-degree relatives with normal cognition (41 parents, children or siblings).

Inclusion criteria $A D$ patients, first-degree relatives of $A D$ patients with normal cognition, age $\geq 40$ years, agreement to participate expressed in written informed consent or, for patients unable to provide this, written consent by legal guardian.

Exclusion criteria Patients with neurological symptoms or signs of other medical conditions (e.g., non-Alzheimer dementia, major depressive disorder, cerebrovascular disease, toxic and metabolic abnormalities); head injury or recent intracranial surgery; patients with another demonstrated comorbidity that causes significant disability (cancer, septic embolism, endocarditis, myeloproliferative disease, creatinine $>3 \mathrm{mg} / \mathrm{dL}$ or $265 \mu \mathrm{mol} / \mathrm{L}$, hyperkalemia $>5.0 \mathrm{mmol} / \mathrm{L}$, chronic or severe liver, kidney, or heart disorders); patients or relatives of patients with histories of alcoholism and/or drug dependence.

\section{Variables included in the study} Clinical diagnosis

Alzheimer disease Persons with clinical disorders consistent with $A D$ who meet the National Institute of Neurological and Communicative Disorders and Stroke and the Alzheimer's Disease and Related Disorders Association (NINCDS-ADRDA) 
Alzheimer criteria (significant and early episodic memory impairment associated with supporting characteristics such as medial temporal lobe atrophy, abnormal cerebrospinal fluid biomarkers, specific patterns in functional and/or metabolic neuroimaging, and autosomal dominant mutation of ApoE).[8-10] Neuropsychological tests were conducted with scores from:

- Montreal Cognitive Assessment (MOCA): <16 points[5]

- Clinical Dementia Rating (CDR): $\geq 1$ point $[18,19]$

- Mini-Mental State Examination (MMSE): $\leq 23$ points[5]

- Global Deterioration Scale (GDS): $\geq 4$ points[20]

Normal cognition (NC) Lack of memory impairment or other cognitive function impairment according to the patient or the relative. Preserved independence in basic activities of daily living and instrumental activities of daily living. Neuropsychological tests were conducted with scores from:

- MOCA: $\geq 26$ points[5]

- CDR: 0 points[18,19]

- MMSE: 27-30 points[5]

- GDS: 1 point[20]

Age Confirmed by the participant's legal identification document (official identity card)

Skin color Based on direct observation and official identity card

Atherogenic risk factors From medical interview

Family history of $A D$ From medical interview

ApoE e4 (Polymerase chain reaction-based genotyping of $A p o E)$ We performed ApoE DNA genotyping using the protocol described by Hixon and Vernier.[21] After this determination, we researched status of participants' ApoE e4 carrier and frequency of the e4 allele. A blood test determined presence of ApoE e4 alleles, recorded as positive or negative.

Cerebral perfusion SPECT We performed SPECT scans after administering the radiopharmaceutical, once participants had been prepared according to internationally standardized protocols.[12]

To perform the SPECT scan, we placed participants in the supine position on a bed in a room with a calm atmosphere and inserted a line into a vein. We left participants to rest, relaxed, in silence, in a dimly lit room, with their eyes closed and without speaking for 10 minutes. Then we administered 740-925 megabecquerels (MBq), at 20-25 millicurie ( $\mathrm{mCi}$ ) of Technium-99m-ethyl cysteinate dimer (99m Tc-ECD) (Tecnonucleo, Argentina) intravenously and waited 10 more minutes in the same conditions before sitting participants up. For the next 30 minutes, we ensured that participants had no intense visual or auditory stimuli. We acquired images 30-45 minutes after radiopharmaceutical administration.

Image acquisition parameters A Nucline Spirit DH-V (Mediso, Hungary) dual-head gamma camera acquired the images. The camera used SPECT acquisition mode with a low-energy highresolution collimator, and a $15 \%$ window centered on the $140 \mathrm{KeV}$ (Kiloelectronvolt) technetium-99m photopeak. Detectors were placed in anterior projection as close as possible to the patient's head. The detector moved automatically along one orbit, rotating $360^{\circ}$, permitting the camera to acquire 180 shots, with a $128 \times 128$ matrix and 1.2 zoom.

\section{Image processing}

Reconstruction Filtered backprojection, with borders right above and below the brain (including the cerebellum).

Filter 4th-order Butterworth, slice frequency 0.25

Attenuation correction Chang's method, coefficient of $0.11 \mathrm{~cm}^{-1}$

Zoom Post-reconstruction variable based on operator's discretion The three axes (sagittal, coronal and transverse) were reoriented so that the transverse slices were parallel to the orbitomeatal line (in the sagittal slice, this line is tangential to the inferior border of the frontal and occipital lobes).

Scan interpretation At least two observers evaluated the SPECT scans, reporting observations independently and subsequently resolving differences by consensus. They evaluated the images in the context of relevant anatomical information provided by CT structural methods. The programs used for image processing contained a database of persons with normal results.

The unprocessed SPECT images ('raw' projections) were reviewed in film mode before CT slices were created, to verify whether the study was complete and to evaluate the presence and degree of movement, abnormal biodistributions, ratio of organ to background activity, and other potential sources of artifacts.

The observers interpreted scans directly from the computer screen, without subtracting background activity. They evaluated scans using continuous color scales and visually evaluated them with slice-by-slice analysis (sagittal, coronal and transverse slices), establishing automatic correlation with the CT. The analysis included 3D images.

Researchers also conducted a color-scale semiquantitative analysis and a quantitative analysis by cerebral region using processing software programs (Mediso, Hungary). This evaluation showed perfusion in the different cerebral lobes and compared one cerebral hemisphere with the other, determining differences between the two.

The results were classified as:

Normal Symmetric distribution of the radiopharmaceutical in one hemisphere with respect to the other. Uniform distribution of the radiopharmaceutical in the cerebral cortex and cerebellum. Basal nuclei with normal and symmetric uptake.

Pathological Focal or diffuse cortical hypoperfusion image, identified in three spatial projections and not corresponding to the interlobar sulci.

Typical $A D$ pattern Evidence of symmetric and predominantly posterior bilateral temporal-parietal cortical hypoperfusion. Enhancement of interhemispheric fissure and sulci suggesting frontal atrophy in the high slice. In advanced stages of the disease, this hypoperfusion included the frontal lobes of the cerebral cortex and the posterior cingulate. Basal nuclei and occipital lobe show normal perfusion.

\section{SPECT stages consistent with AD}

Stage 1 Any abnormality of diffuse unilateral posterior temporalparietal perfusion. 
Stage 2 Symmetric and predominantly posterior bilateral temporal-parietal cortical hypoperfusion, of moderate intensity.

Stage 3 Symmetric and predominantly posterior bilateral temporal-parietal cortical hypoperfusion, of severe intensity and extending to the frontal lobe, associated with generalized cerebral cortex thinning.

Data compilation and analysis We evaluated 128 clinical charts and medical reports of patients and relatives who provided written informed consent. Data were coded on an Excel spreadsheet, maintaining confidentially and anonymity, and entered into double-entry tables with participants' general characteristics. We divided patients into two study groups (patients with $A D$ and first-degree relatives with NC), determined the number and percentage of patients based on SPECT results, and created graphs with the data obtained. We obtained the absolute and relative frequencies of patients with $A D$ based on the three clinical stages of the disease.

We created cross-classification tables to explore the association between clinical classification and the presence or absence of ApoE e4 with the results of the cerebral perfusion SPECT.

Ethical considerations The ethics committees of all participating institutions (Isotope Center, Cuban Neuroscience Center, and the Artemisa Provincial Health Department Comprehensive Memory Disorder Community Care Service) analyzed and approved the research. We encrypted participant data for analysis. All participants (patients or their legal guardians, and their relatives) gave informed consent in writing.

\section{RESULTS}

AD patient group Included in this group were 60 women and 27 men. The mean age was 74 , and skin color was classified in $60.9 \%(53 / 87)$ as white and $39.1 \%(34 / 87)$ as mestizo (according to categories established by the Cuban census). The atherogenic risk factors in the patients were diabetes mellitus in $62 \%(54 / 87)$, dyslipidemia in $50 \%$ (44/87) and arterial hypertension in $31 \%$ (27/87). A family history of $A D$ was reported in $62 \%$ (54/87) of patients.

Cerebral perfusion SPECT Evaluation showed a typical AD pattern (bilateral posterior temporal-parietal hypoperfusion) in $77 \%(67 / 87)$ of patients. A lower percentage, $12.7 \%(11 / 87)$, showed mild or unilateral perfusion changes consistent with the initial stage of AD. We observed mild-to-moderate focal hypoperfusion patterns consistent with vascular disease in $5.7 \%(5 / 87)$ of patients (individuals with an initial diagnosis of $A D)$. In only $3.4 \%(3 / 87)$ of patients did we observe frontal hypoperfusion with good perfusion in the rest of the cerebral cortex (pattern consistent with frontal dementia), and we observed in 1.2\% (1/87) normal cerebral perfusion (Figure 1).

Cerebral SPECT results showed changes consistent with $A D$ in 78 patients-typical $A D$ pattern or minimal unilateral perfusion changes (Figure 2). Most $A D$ patients, $51.3 \%$ $(40 / 78)$, were in stage 2 of the disease (moderate); $35.9 \%$ (28/78) were in stage 1 (mild); and $12.8 \%$ (10/78) were in stage 3 (advanced). The bottom of the figure shows the cross-
Figure 1: Patients with clinical diagnosis of Alzheimer disease group $(\mathbf{n}=87)$ : SPECT cerebral perfusion results

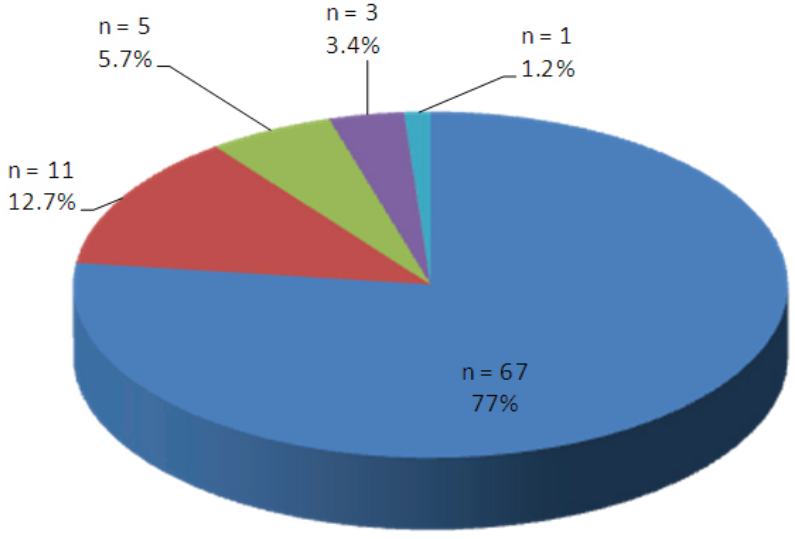

- Typical AD pattern Mild and/or unilateral changes in cerebral perfusion - Focal hypoperfusion Erontal hypoperfusion Normal perfusion

Figure 2: Patients by Alzheimer Disease stages according to cerebral SPECT $(n=78)$

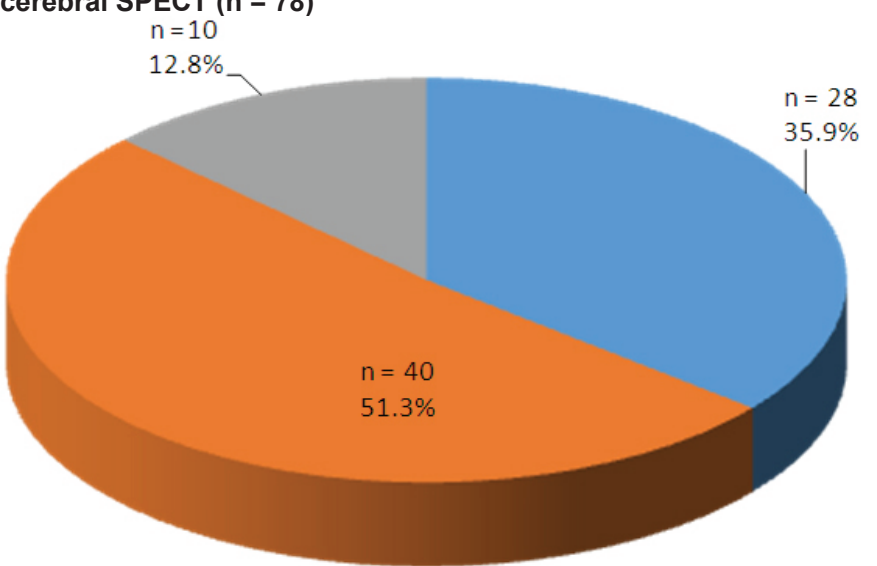

- Stage 1 Stage 2 Stage 3

SPECT: Single photon emission computed tomography

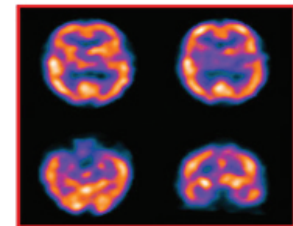

Alzheimer stage1

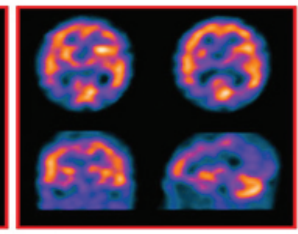

Alzheimer stage 2

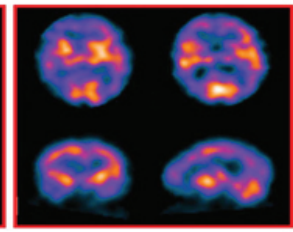

Alzheimer stage 3
Stage 1: Any abnormality of diffuse unilateral posterior temporalparietal perfusion

Stage 2: Symmetric and predominantly posterior bilateral temporalparietal cortical hypoperfusion, of moderate intensity

Stage 3: Symmetric and predominantly posterior bilateral temporalparietal cortical hypoperfusion, of severe intensity and extending to the frontal lobe, associated with generalized cerebral cortex thinning

sections of cerebral perfusion SPECT with typical patterns for each of the three AD stages.

In most patients, clinical diagnoses matched SPECT results. However, $28.7 \%$ (25/87) of patients received a different classification of disease stage and disease diagnosis after cerebral perfusion results were considered; five patients with vascular dementia and three patients with frontal-temporal dementia. 
Figure 3: First-degree relatives with normal cognition group $(n=41)$ : SPECT cerebral perfusion results

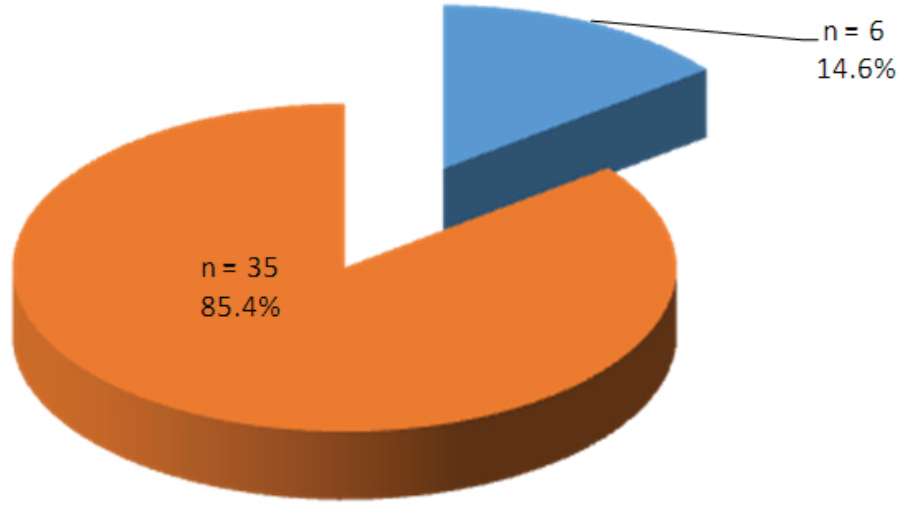

Altered perfusion

Normal perfusion

SPECT: Single photon emission computed tomography

Figure 4: Transverse slices of cerebral perfusion SPECT of a 67-year-old woman (relative with normal cognition), positive ApoE e4
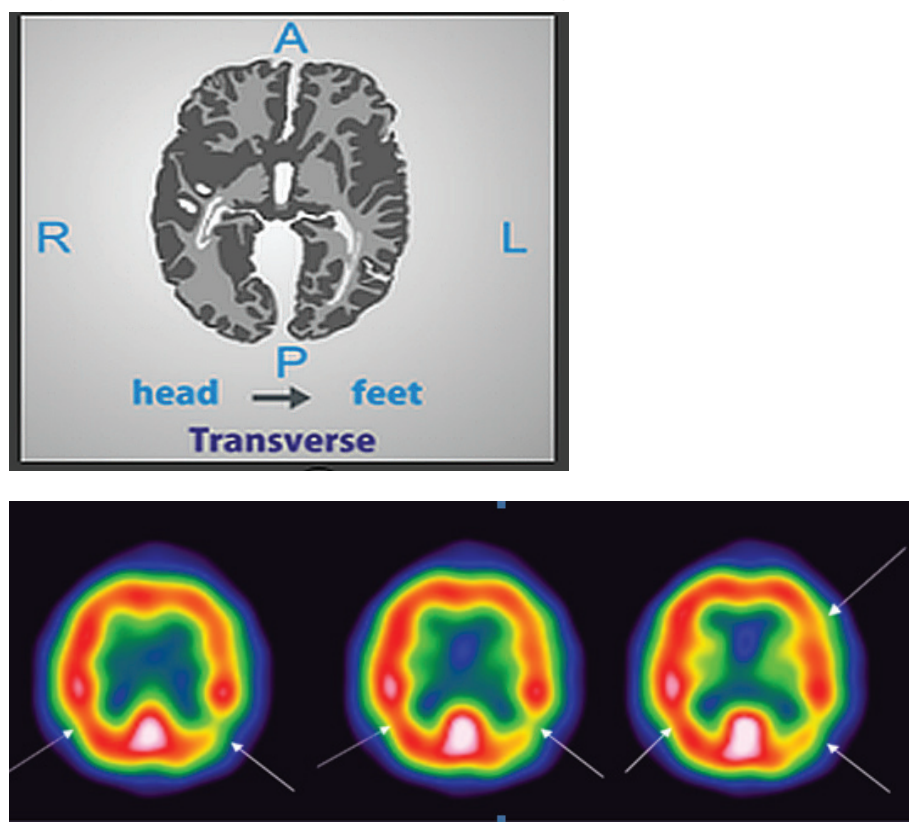

ApoE e4: APOE genotype

The arrows point to regions with mild bilateral temporal-parietal hypoperfusion, more severe in the left hemisphere. Images also show mild diffuse hypoperfusion in the left frontal lobe. A: Anterior; L: Left; P: Posterior; R: Right

First-degree relatives group This group included 28 women and 13 men. The mean age was 56 , and skin color in $60 \%$ $(25 / 41)$ of participants was classified as white, and as mestizo in $40 \%(16 / 41)$ of patients. Atherogenic risk factors were diabetes mellitus in 58\% (24/41), dyslipidemia in 41\% (17/41), and arterial hypertension in $29 \%(12 / 41)$. All participants (41/41) had a family history of $A D$.

Cerebral perfusion SPECT This showed regional cerebral perfusion abnormalities in $14.6 \%$ (6/41) of evaluated participants (Figure 3 ). We found mild bilateral temporalparietal hypoperfusion in $7.1 \%(3 / 41)$, unilateral mild temporal and parietal hypoperfusion in $4.8 \%(2 / 41)$, and a diffuse perfusion defect of the right frontal lobe in $2.4 \%(1 / 41)$. None of these cases had a result consistent with normal variants. Changes in cerebral perfusion could be predictors of AD.

The cerebral perfusion SPECT of a first-degree relative with normal cognition (a woman aged 67 with hypertension and type 2 diabetes, daughter of a patient with $A D$ ) showed mild bilateral temporal-parietal hypoperfusion, more severe in the left hemisphere (Figure 4). The images also revealed mild diffuse hypoperfusion in the left frontal lobe. Before the study, this relative had not reported any symptoms suggestive of $A D$.

Cerebral perfusion SPECT and ApoE e4 We conducted a cross-comparison between the cerebral perfusion SPECT results and presence or absence of ApoE e4 in blood for both groups evaluated in the study (patients with AD and first-degree relatives with $\mathrm{NC}$ ) (Table 1). We observed a link between ApoE e4 presence in blood and alterations in perfusion reported by the SPECT. Of participants with a typical AD pattern in the cerebral SPECT, $76.5 \%$ had positive ApoE e4. In the AD patients' group, ApoE e4 was also positive in $45.5 \%$ of cases with mild and/or unilateral changes in cerebral perfusion. All first-degree relatives with SPECT perfusion abnormalities had positive ApoE e4. All participants who had frontal and focal hypoperfusion, with normal perfusion in parietal and temporal regions, had negative ApoE e4.

\section{DISCUSSION}

Neuroimaging techniques have made remarkable advances, specifically in the areas of AD diagnosis, prognosis and predicting response to treatment. The most frequently used techniques include MRI, PET, and cerebral perfusion SPECT. $[12,15,22,23]$

Advances in neurosciences have raised awareness of the following: 1) existence of patients who have diffuse amyloid plaques typical of AD without dementia; 2) that characteristic disease pathology can be accompanied by atypical clinical manifestations; and 3) abnormal pathology occurs years before the first clinical symptoms appear-dementia is the final stage of the disease. It is therefore possible to differentiate two AD stages: a first preclinical, asymptomatic stage; and a second, symptomatic stage, from the appearance of the first cognitive manifestations to the more advanced phases of dementia.[8]

Nuclear medicine studies such as SPECT and PET could be important in supporting early AD diagnosis.[8,10] Currently, the sensitivity and specificity values of PET scan metabolic imaging are higher than those of SPECT. PET scans are able to detect glucose metabolism in brain cells as well as deposits of beta-amyloid plaques and tau proteins using specific radiopharmaceuticals. Multiple clinical trials are being conducted with these specific markers.[24] However, high cost, limited availability in most countries, and the need to rely on radioactive isotopes with a short or ultra-short half lives have limited the use of PET scans as a screening method on asymptomatic patients.[11]

Although PET and SPECT scans use different tracers and instruments, the principles of interpretation and underlying neurobiological processes are similar. There is a confirmed association between perfusion SPECT results and PET results, 
Table 1: Comparison of cerebral perfusion SPECT results and presence or absence of ApoE e4 in patients and family members

\begin{tabular}{|c|c|c|c|c|c|}
\hline \multirow[b]{2}{*}{ Group } & \multirow{2}{*}{\multicolumn{2}{|c|}{$\begin{array}{c}\text { Cerebral SPECT } \\
\text { result }\end{array}$}} & \multicolumn{3}{|c|}{ ApoE e4 } \\
\hline & & & $\begin{array}{l}\text { ApoE e4 } \\
\text { Positive }\end{array}$ & $\begin{array}{l}\text { ApoE e4 } \\
\text { Negative }\end{array}$ & Total \\
\hline \multirow{10}{*}{$\begin{array}{l}\text { Alzheimer } \\
\text { patients }\end{array}$} & \multirow{2}{*}{$\begin{array}{l}\text { Typical AD } \\
\text { pattern }\end{array}$} & $\mathrm{n}$ & 52 & 16 & 68 \\
\hline & & $\%$ & 76.5 & 23.5 & 100 \\
\hline & \multirow{2}{*}{$\begin{array}{l}\text { Minimal changes } \\
\text { in perfusion }\end{array}$} & $\mathrm{n}$ & 5 & 6 & 11 \\
\hline & & $\%$ & 45.5 & 54.5 & 100 \\
\hline & \multirow{2}{*}{$\begin{array}{l}\text { Focal } \\
\text { hypoperfusion }\end{array}$} & $\mathrm{n}$ & 0 & 5 & 5 \\
\hline & & $\%$ & 0 & 100 & 100 \\
\hline & \multirow{2}{*}{$\begin{array}{l}\text { Frontal } \\
\text { hypoperfusion }\end{array}$} & $\mathrm{n}$ & 0 & 3 & 3 \\
\hline & & $\%$ & 0 & 100 & 100 \\
\hline & \multirow{2}{*}{ Normal perfusion } & $\mathrm{n}$ & 1 & 0 & 1 \\
\hline & & $\%$ & 100 & 0 & 100 \\
\hline \multirow{4}{*}{$\begin{array}{l}\text { First-degree } \\
\text { relatives }\end{array}$} & \multirow{2}{*}{$\begin{array}{l}\text { Minimal changes } \\
\text { in perfusion }\end{array}$} & $\mathrm{n}$ & 6 & 0 & 6 \\
\hline & & $\%$ & 100 & 0 & 100 \\
\hline & \multirow{2}{*}{ Normal perfusion } & $\mathrm{n}$ & 9 & 25 & 34 \\
\hline & & $\%$ & 26.5 & 73.5 & 100 \\
\hline \multirow{2}{*}{ Total } & & $\mathrm{n}$ & 73 & 55 & 128 \\
\hline & & $\%$ & 57 & 43 & 100 \\
\hline
\end{tabular}

AD: Alzheimer disease ApoE e4: APOE genotype

SPECT: Single photon emission computed tomography

and in degenerative diseases, the two have shown links between cerebral blood flow and metabolism.[11] Regional cerebral blood flow changes are an indirect manifestation of changes in oxygen use and metabolism; therefore, SPECT scans can identify patterns that are repeated in PET scans. SPECT scans also have the added advantage of being cheaper and more widely available.[11]

Cuba's Ministry of Public Health has recently outlined a plan to optimize medical equipment in the country, increasing availability of cerebral SPECT tests and facilitating use of the perfusion test to be used in clinical practice. However, as far as we know, ours is the first study in Cuba related to cerebral SPECT tests on AD. SPECT not only evaluates cerebral blood flow, but also provides valuable information to support diagnosis in early stages of $A D$ and provides a useful tool for minimally invasive research on human cognition.[12,13]

This study describes the results of evaluating patients with $A D$ and their relatives using cerebral SPECT tests; shows the usefulness of this technique in diagnosis, progression, and differential diagnosis of the patients; provides information about usefulness of SPECT tests for studying first-degree relatives; and compares the SPECT results with clinical evaluation and ApoE e4 tests.

AD Patient group Characteristics were similar to those reported in other Cuban studies of AD patients. In our study, the mean age was $\geq 65$ years, and participants were predominantly women. Many studies show a greater prevalence of $A D$ in women and show that most long-term caregivers of patients are women, which indicates that the disease doubly affects women, who therefore require greater care and support.[2,4]

Most patients evaluated were white, which is similar to studies by other authors that report lower prevalences of AD in persons of African descent.[2] Ethnicity is also related to predispositions for cognitive disorders, and ApoE e4 has been shown to vary based on ethnicity, with lower expression in persons of African descent.[2]

We found three AD risk factors-diabetes mellitus, dyslipidemia and high blood pressure-in a high percentage of patients, similar to findings of other authors who posit that risk factors associated with heart disease and stroke can also increase AD risk.[27] They argue that high blood pressure can damage blood vessels in the brain, resulting in abnormalities in regions important for decision-making, memory and verbal skills, therefore affecting AD progression.[27] These authors also suggest that high cholesterol can inhibit the blood's ability to eliminate unwanted proteins (beta-amyloid plaques and tau) from the brain. Additionally, growing evidence supports a link between $A D$ and type 2 diabetes, since higher glucose levels in the blood can damage the brain and contribute to the $A D$ progression.[27]

A high percentage of patients had a family history of AD. Studies have shown that persons have a greater chance of $A D$ when their father, mother, brother or sister has had the disease. [28] The risk increases if more than one relative has had the disease. When diseases such as $A D$ tend to recur in families, environmental as well as hereditary factors may be important. [28]

Cerebral SPECT images showed a typical AD pattern in a high percentage of patients $(77 \%)$. A lower proportion of patients showed patterns consistent with cerebrovascular disease and frontal dementia, and in one patient the cerebral perfusion result was normal. These results confirm the value of SPECT for supporting $A D$ diagnosis generally and for establishing a differential diagnosis between dementia, depressive disorders, psychiatric disorders and other diseases that could be initially confused with AD.[29-31]

SPECT also helps classify patients based on disease progression stages, identify patients' functional status, and thus evaluate possible interventions and drug therapies. While disease-stage classification reported by medical orders sent to nuclear medicine usually matched SPECT findings, a number of patients $(n=25)$ were diagnosed in a different stage based on imaging results. Most of them were in the initial disease stage, when intervention to slow progression is more useful.

Many studies conducted internationally also show that SPECT brain imaging has become a valuable tool for evaluating dementia,[29-32] with the aim of better understanding functional impairments associated with the disease and improving diagnostics. The insidious onset of $A D$ is often confused with normal aging processes. In these stages, the SPECT technique plays a fundamental role.[12,13,29-31]

SPECT findings characteristic of AD are bilateral temporalparietal hypoperfusion with limited participation in sensorymotor and occipital regions. This perfusion pattern can distinguish $A D$ patients from those with normal results, with a sensitivity of $70 \%-100 \%$, a specificity of $87 \%-100 \%$, and a diagnostic confidence of $98 \%$.[13] In advanced stages, in 
addition to temporal-parietal hypoperfusion, blood flow is reduced in the frontal, parietal and occipital cortex areas.[13]

Some 25 years ago, McMurdo used SPECT brain imaging to assess 26 patients with a clinical diagnosis of AD: 15 showed bilateral temporal-parietal hypoperfusion, 4 normal perfusion, 4 signs of ischemic episodes, and 3 frontal hypoperfusion with normal perfusion of the temporal-parietal cerebral cortex.[32] These results were similar to our study findings.

Other research in $A D$ patients has revealed an association between cerebral blood flow patterns and the histopathological findings typical of the disease. Authors of a prospective study estimated the predictive value of SPECT in AD diagnosis and found that a patient with memory loss and a normal SPECT has a $19 \%$ probability of developing $A D$. In comparison, the probability of developing $A D$ in patients with memory loss and bilateral temporal-parietal hypoperfusion increases to $82 \%$. Patients without perfusion defects in the SPECT have a very low probability of getting AD.[29]

Several studies have used SPECT for differential diagnosis in dementia and have described some characteristic perfusion patterns. Dementia of vascular origin shows single or multiple areas of hypoperfusion with wedge-shaped or semicircular appearances in any region of the cerebral cortex. In Parkinson disease, the results are variable as cerebral regional blood flow changes are based on L-Dopa levels and on the presence or absence of dementia (and, if present, its severity). Early stages exhibit hypoperfusion in the caudate nuclei and thalamus, which, among other areas, are involved in disease progression. Studies have described hypoperfusion of the basal ganglia, specifically in the caudate nuclei, in patients with Huntington disease. Patients with Creutzfeldt-Jakob disease exhibit cortical hypoperfusion of the frontal and parietal lobes. Patients with depression show decreased cerebral blood flow in the prefrontal cortex and in the limbic and paralimbic systems.[29]

First-degree relatives with normal cognition Several general characteristics of this group are similar to those of the patient group. The mean age in this case was $<65$, which was expected, since the group included the children of some study patients. These results once again show the effect of age on symptom onset and imply that cerebral perfusion assessment could assist with early detection.

Like the patient group, this group was predominantly women. The fact that many patients arrived with their female caregivers (relatives who also participated in the study) could explain this finding. Due to family connections with the patients, participants in this group were predominantly white. The most common atherogenic risk factors were also diabetes, dyslipidemia, and hypertension, but in a smaller percentage than the patient group.

Approximately $15 \%$ of the relatives showed changes in cerebral perfusion consistent with initial AD stages. Tests confirmed that $100 \%$ of these subjects had positive ApoE e4, which increases risk. These results enabled early screening of a group with a high probability of developing $A D$, who can then be provided with early clinical and therapeutic support to slow the onset of disease.
SPECT brain imaging and ApoE e4 There was a strong association between SPECT results and presence or absence of ApoE e4 for the 128 study subjects (Table 1 ). More than $70 \%$ of $A D$ patients with typical AD patterns in the cerebral SPECT had a positive ApoE e4, which matched reports by other authors.[16] All the relatives with changes in cerebral perfusion had positive ApoE e4.

For years, the e4 allele of ApoE has been associated with increased risk of dementia. The ApoE gene seems to be a risk factor for late-onset $A D$. There are three forms of this gene: ApoE e2, ApoE e3 and ApoE e4. Even though inheriting ApoE e4 increases AD risk, APO e2 provides substantial protection against the disease. Some research focuses on the association between these two forms of ApoE and Alzheimer disease.[16,33]

Previous studies confirm that the ApoE e4 allele's involvement in cerebral blood flow may have therapeutic implications for AD patients. Although presence of the ApoE e4 allele increases $A D$ risk and decreases the mean age of onset, the association between positive ApoE e4 and cerebral blood flow is disputed. Some authors suggest that this association may depend on the patient's age and AD stage.[34]

This study found an association between presence of ApoE e4 and decreased cerebral perfusion, but additional studies are needed on potential associations between cerebral blood flow, ApoE, vascular risk factors, and neuroimaging markers of cerebrovascular disease to provide more information about ApoE interaction and neurovascular changes in brain function in older adults at risk for dementia.

Despite the limitations of this study-small sample size, lack of comparison with anatomic and metabolic imaging results such as MRI and PET, and lack of a control group of healthy persons properly balanced regarding age and other relevant covariates - the research is important because this is the first time that a group of patients with $A D$ and first-degree relatives in a community have been jointly evaluated in Cuba using SPECT and the ApoE e4 test. The study showed the usefulness of cerebral SPECT, which is not often used to evaluate AD patients in our setting and revealed the usefulness in patients and populations with identified risk factors (first-degree relatives with NC who are ApoE e4 positive) for timely diagnosis and subsequent intervention strategies at the primary care level.

\section{CONCLUSIONS}

The cerebral perfusion test allowed us to confirm the diagnosis of Alzheimer disease, classify the disease stage, and differentiate between the types of dementia. The SPECT brain imaging showed perfusion changes in several asymptomatic first-degree relatives with positive ApoE e4, which could be predictors of disease. The technique was useful for evaluating both patients and relatives, who received an early warning of their risk of developing the disease.

\section{ACKNOWLEDGMENTS}

We conducted this study as part of a project with the Cuban Agency of Nuclear Energy and Advanced Technology (AENTA) program: PNUOLU/3-1/1/2019-2020: "Developing skills in nuclear medicine departments for clinical use of functional neuroimaging in Alzheimer's disease." - 1 - 


\section{REFERENCES}

1. Harvard Health. Recognizing Alzheimer's disease. Early Warning Signs and Diagnosis [Internet]. Santa Monica: Help Guide.org; 2020 [cited 2020 Apr 28]; [about 9 p.]. Available at: https:// www.helpguide.org/harvard/recognizing-and -diagnosing-alzheimers.htm

2. Llibre Guerra JJ, Díaz Marante JP, Rodríguez Salgado AM, Peñalver Al, Guartazaca Guerrero EP, Rousseaux Mola E, et al. Determinantes del estado de salud de la población y su influencia en el desarrollo de los trastornos cognitivos. Rev Cubana Salud Pública. 2018 Jan-Mar;44(1):141-52. Spanish.

3. National Medical Records and Health Statistics Division (CU). Anuario Estadístico de Salud 2019 [Internet]. Havana: Ministry of Public Health (CU); 2019 [cited 2020 May 21]. p. 39-43. Available at: https://files.sld.cu/bvscu ba/files/2020/05/Anuario-Electr\%c3\%b3nicoEspa\%c3\%b1ol-2019-ed-2020.pdf. Spanish.

4. Llibre Rodríguez JJ. Envejecimiento y demencias: implicaciones para la comunidad científica, la salud pública y la sociedad cubana. Rev An Acad Ciencias Cuba. 2012;2(2):1-18. Spanish.

5. Romero Cabrera AJ, Fernández Casteleiro E, López Argüelles J, Suz Piña JD, Cordero Jiménez JR. Actualización en diagnóstico y manejo de las demencias. Aportes prácticos en nuestro contexto. Rev Finlay [Internet]. 2012 [cited 2020 May 8];2(1):76-87. Available at: http://revfinlay. sld.cu/index.php/finlay/article/view/76Quijano. Spanish.

6. Alzheimer's Disease International. World Alzheimer Report 2015. The Global Impact of Dementia. An analysis of prevalence, incidence, cost and trends [Internet]. London: Alzheimer's Disease International; 2015 [cited 2020 May 8]. 82 p. Available at: https://www.alz.co.uk/ research/world-report-2015

7. Llibre Rodríguez JJ, Gutiérrez Herrera RF. Demencias y enfermedad de Alzheimer en América Latina y el Caribe. Rev Cubana Salud Pública. 2014;40(3):378-87. Spanish.

8. López-Álvarez J, Agüera-Ortiz LF. Nuevos criterios diagnósticos de la demencia y la enfermedad de Alzheimer: una visión desde la psicogeriatría. Psicogeriatría. 2015 Mar;5(1):3-14. Spanish.

9. Rojo Martínez E. Enfermedad de Alzheimer. Nuevos criterios diagnósticos e implicaciones en la práctica clínica. An Real Acad Med Cir Vall. 2014;51:67-76. Spanish.

10. Dubois B, Feldman HH, Jacova C, Dekosky ST, Barberger-Gateau P, Cummings J, et al. Research criteria for the diagnosis of Alzheimer's disease: revising the NINCDS-ADRDA criteria. Lancet Neurol. 2007 Aug;6(8):734-46.

11. Jiménez JF, Carril Carril JM. La neuroimagen molecular en las demencias degenerativas. Rev Española Med Nucl Imagen Mol. 2013 Sep-Oct;32(5):301-9. Spanish.

12. Juni JE, Waxman AD, Devous MD, Tikosfky RS, Ichise M, Van Heertum RL, et al. Procedure Guideline for Brain Perfusion SPECT Using 99mTc Radiopharmaceuticals 3.0*[Internet]. Virginia: Society of Nuclear Medicine and Molecula Imaging; 2009 [cited 2020 Apr 28]. 5 p. Available at: https://s3.amazonaws.com/rdcms-snmmi/files/ production/public/docs/Brain_SPECT_Guideline 2003.pdf

13. Rojas G, Ladrón de Guevara D, Jaimovich R. Neuroimágenes en demencias. Rev Med Clin Condes. 2016;27(3):336-56. Spanish.

14. Teipel S, Drzezga A, Grothe MJ, Barthel H, Chételat $\mathrm{G}$, Schuff $\mathrm{N}$, et al. Multimodal imaging in Alzheimer's disease: validity and usefulness for early detection. Lancet Neurol. 2015 Oct;14(10):1037-53.

15. Narayanan L, Murray AD. What can imaging tell us about cognitive impairment and dementia? World J Radiol. 2016 Mar 28;8(3):240-54

16. Lopera F, Siegert I, Arcos-Burgos M, Ríos A. Alteraciones en el SPECT cerebral antes del inicio de la enfermedad de Alzheimer precoz producida por la mutación E280A de la PS1. Acta Med Colomb. 2000 May-Jun;25(3):144-9.

17. Bosch-Bayard R, Llibre-Rodríguez JJ, Fernández-Seco A, Borrego-Calzadilla C, CarrascoGarcía MR, Zayas-Llerena T, et al. Cuba's Ssrategy for Alzheimer disease and dementia syndromes. MEDICC Rev. 2016 Oct;18(4):9-13.

18. Morris JC. The Clinical Dementia Rating (CDR) current version and scoring rules. Neurology. 1993 Nov;43(11):2412-4

19. Williams MM, Storandt M, Roe CM, Morris JC. Progression of Alzheimer disease as measured by Clinical Dementia Rating sum of boxes scores. Alzheimers Dement. 2013 Feb;9(1 Suppl):S39-44.

20. Custodio N, Becerra-Becerra Y, Alva-Díaz C, Montesinos R, Lira D, Herrera-Pérez E, et al. Validación y precisión de la Escala de Deterioro Global (GDS) para establecer severidad de demencia en una población de Lima. Rev CES Med. 2017 Jan-Jun;31(1):14-26. Spanish.

21. Hixson JE, Vernier DT. Restriction isotyping of human apoli $E$ by gene amplification and cleavage with Hhal. J Lipid Res. 1990;31:545-8. Spanish.

22. European Association of Nuclear Medicine (EANM) [Internet]. Vienna: European Association of Nuclear Medicine (EANM); c2020. Publications. Technologist's guide. Brain Imaging; 2016 [cited 2020 May 11]. Available at: https://www .eanm.org/publications/technologists-guide/ brain-imaging/

23. Soricelli A. Nuclear medicine resources manual IAEA [Internet]. Vienna: International Atomic Energy Agency; 2006 [cited 2020 May 11]. 532 p. Available at: https://www.iaea.org/publica tions/7038/nuclear-medicine-resources-manual

24. La Joie Renaud, Visani AV, Baker SL, Brown JA, Bourakova Viktoriya, Cha J. Prospective longitudinal atrophy in Alzheimer's disease correlates with the intensity and topography of baseline tauPET. Sci Translat Med. 2020 Jan 1;12(524):5732. DOI: 10.1126 / scitransImed.aau5732.

25. Ecuador Ministry of Health, Health Sector Stratetig Development Plan, Department of Health Economics Estudio de costos del equipo de diagnóstico PET-CT [Internet]. Washington, D.C.: Pan American Health Organization, RedETSA; 2012 [cited 2020 May 11]. Available at: https://sites. bvsalud.org/redetsa/brisa/resource/?id=biblioref .referencesource.905780. Spanish.

26. Hlatky MA, Shilane D, Hachamovitch R, DiCarli MF; SPARC Investigators. Economic outcomes in the study of myocardial perfusion and coronary anatomy imaging roles in coronary artery disease registry. J Am Coll Cardiol. 2014 Mar 18;63(10):1002-8. DOI:10.1016/j.jacc .2013.11.038.

27. Santos CY, Snyder PJ, Wu WC, Zhang M, Echeverría A, Alber J. Pathophysiologic relationship between Alzheimers's disease, cerebrovascular disease, and cardiovascular risk: a review and synthesis. Alzheimeers Dement (Amst). 2017 Feb 9;7:69-87.

28. Padrón Pérez N, Gra Menéndez S, Llibre Rodríguez JJ. Presenilinas, Apo E y Enfermedad de Alzheimer. Rev Cubana Invest Bioméd. 2002 Oct-Dec;21(4):262-9. Spanish.

29. Castañeda M, García JC, García JA, Gutierrez LM, Ostrosky F. Actualización por temas. SPECT cerebral y enfermedad de Alzheimer: una revisión. Salud Mental. 2000 Feb;23(1):39-45. Spanish

30. Valotassiou V, Malamitsi J, Papatriantafyllou J, Dardiotis E, Tsougos I, Psimadas D, et al. SPECT and PET imaging in Alzheimer's disease. Ann Nucl Med. 2018 Nov;32(9):583-93.

31. Yeo JM, Lim X, Khan Z, Pal S. Systematic review of the diagnostic utility of SPECT imaging in dementia. Eur Arch Psychiatry Clin Neurosci. 2013 Oct;263(7):539-52

32. McMurdo M, Grant DJ, Kennedy NS, Gilchrist J, Findlay D, McLenna JM. The value of HMPAO SPECT scanning in the diagnosis of early Alzheimer's disease inpatients attending a memory clinic. Nucl Med Commun. 1994 Jun;15(6):405-9.

33. Samper Noa JA, Llibre Rodríguez JJ, Sánchez Catases C, Sosa Pérez S. El deterioro cognitivo leve. Un paso antes de la enfermedad de Alzheimer. Rev Haban Cienc Méd. 2011 Jan -Mar;10(1):27-36. Spanish.

34. Wierenga CE, Clark LR, Dev SI, Shin DD, Jurick $\mathrm{SM}$, Rissman RA, et al. Interaction of age and APOE genotype on cerebral blood flow at rest. J Alzheimers Dis. 2013;34(4):921-35.

\section{THE AUTHORS}

Yamilé Peña-Quián (corresponding author: yamilepq@infomed.sld.cu; yamicuba2014@ gmail.com), physician specializing in internal medicine and nuclear medicine, with a doctorate in medical sciences. Senior researcher, Clinical Research Department, Isotope Center, Havana, Cuba. https://orcid.org/0000-0002 $-6598-6099$

Saily Sosa-Pérez, internist with a master's degree in satisfactory longevity. Adjunct researcher, Artemisa Provincial Health Department Comprehensive Memory Disorder Com- 
munity Care Service, Artemisa, Cuba. https:// orcid.org/0000-0002-8221-332X

Department, Cuban Neuroscience Center, Havana, Cuba. https://orcid.org/0000-0002-3958 $-183 X$

Juan Felipe Batista-Cuéllar, physician specializing in internal medicine and nuclear medicine. Clinical Research Department, Isotope Center, Havana, Cuba. https://orcid.org/0000-0001 $-9420-4255$

Chryslaine Rodríguez-Tanty, chemist with a doctorate in organic chemistry. Senior researcher and head of the Experimental Neuroscience
Leonel Alberto Torres-Aroche, nuclear physicist with a doctorate in pharmaceutical sciences. Senior researcher, Clinical Research Department, Isotope Center, Havana, Cuba. https:// orcid.org/0000-0001-5019-1915

Elvia Luz-Sánchez, nurse with a master's degree in intensive care. Clinical Research
Department, Isotope Center, Havana, Cuba. https://orcid.org/0000-0001-6870-5339

Susana Romero-Collado, nurse with a master's degree in intensive care Clinical Research Department, Isotope Center, Havana, Cuba. https://orcid.org/0000-0003-2616-8349

Submitted: June 22, 2020

Approved for publication: January 14, 2021

Disclosures: None 